\title{
COVID-19: Impflicht für Arbeitnehmer im medizinischen Bereich?
}

Klara Bothe

\begin{abstract}
Nachdem inzwischen die Mehrzahl des medizinischen Personals gegen COVID-19 geimpft oder zumindest impfbereit ist, ist zu erörtern, ob Arbeitnehmer zu einer Schutzimpfung verpflichtet werden können. Dürfen Arbeitgeber Nachweise einer Impfung verlangen und drohen arbeitsrechtliche Konsequenzen aus einer Impfverweigerung? Aktuell besteht keine allgemeine gesetzliche COVID-19-Impfpflicht. Jede verpflichtende Impfung ist ein Eingriff in das Grundrecht auf körperliche Unversehrtheit und auf informationelle Selbstbestimmung. Dieser Grundrechtseingriff bedarf der Abwägung im konkreten Einzelfall.
\end{abstract}

\section{Impfpflicht aus Arbeitsvertrag}

Unabhängig vom Bestehen einer gesetzlichen Impfpflicht kann eine solche vertraglich i.F.v. Tarifverträgen oder Arbeitsverträgen vereinbart werden. Den Tarifvertragsparteien steht es grundsätzlich frei Regelungen zu treffen, die die körperliche Unversehrtheit betreffen. Diese Regelungen sind jedoch unzulässig, wenn sie die Grundrechte der Arbeitnehmer unangemessen beschränken.

\section{Merke}

Die Anordnung über die Durchführung von ärztlichen Kontrollmaßnahmen ist damit nicht per se unzulässig.

Gleichwohl sind Arbeitgeber nicht berechtigt, uneingeschränkt ärztliche Untersuchungen ihrer Arbeitnehmer zu verlangen, die sie für sachdienlich halten [1]. Voraussetzung ist, dass eine gebotene Abwägung zwischen den Arbeitgeber- und den Arbeitnehmerinteressen entsprechend dem Verhältnismäßigkeitsgrundsatz erfolgt (stRspr. BAG NZA 2019, 619; NJW 2011, 3319). Dabei sind die Rechte des Arbeitgebers auf unternehmerische Freiheit, auf den eingerichteten und ausgeübten Gewerbebetrieb und der allgemeinen Handlungsfreiheit dem Grundrecht auf körperliche Unversehrtheit aus Art. 2 Abs. 2 GG und dem Recht auf informationelle Selbstbestimmung aus Art. 2 Abs. 1 i. V.m. 1 Abs. 1 GG des Arbeitnehmers im konkreten Einzelfall gegenüberzustellen. Dies gilt besonders für die Festlegung gewisser Verhaltensnormen. Ein legitimes Arbeitgeberinteresse ist der Schutz vor der Virusansteckung und Verbreitung innerhalb der Mitarbeiter und den daraus resultierenden möglichen Arbeits- und Betriebsausfällen. Auch besteht i. d. R. das Interesse, durch eine lückenlose Impfung die kostspieligen Hygiene-, Abstands- und Sicherheitsmaßnahmen zu reduzieren. Allerdings hat eine Schutzimpfung rein präventive Wirkung und dient nicht der Gefahrenabwehr. Das Nichtvorliegen einer Impfung beeinträchtigt die Erbringung der Arbeitsleistung nicht. Indes trägt der Arbeitgeber durch die Anordnung einer Impfung seiner Schutzpflicht gegenüber Risikogruppen innerhalb des Betriebs Rechnung.

Gegenüberzustellen ist das Recht des Arbeitnehmers, frei über seine körperliche Unversehrtheit und seine persönliche Lebensgestaltung zu entscheiden. Eine Impfung bedeutet einen erheblichen Eingriff in die körperliche Unversehrtheit, indem eine fremde Substanz in den Körper injiziert wird. Aufgrund der grundrechtlichen Bedeutung von Art. 2 Abs. 2 GG und Art. 2 Abs. 1 i. V. m. 1 Abs. 1 GG ist eine Impfpflicht unterschiedslos für alle Arbeitnehmer ohne Abwägung im Einzelfall unverhältnismäßig und damit unzulässig.

\section{Merke}

In Arbeitsverträgen kann eine Impfpflicht im medizinischen Tätigkeitsfeld grundsätzlich vereinbart werden.

Eine solche Klausel unterliegt der AGB-Kontrolle nach $\S \S$ 305 ff. BGB und wird wohl zulässig sein, da die Einhaltung von Präventionsmaßnahmen und die Vermeidung von Infektions- und Gesundheitsrisiken in diesem Bereich allgemein üblich sind. Für Arbeitnehmer außerhalb des medizinischen Personals kann eine entsprechende Klausel überraschend und unzulässig sein [2], da eine Schutzimpfung i. d. R. kein dienstliches Verhalten darstellt.

\section{Impflicht aus Direktionsrecht und Treuepflicht}

Dem Arbeitgeber steht ein Weisungsrecht gegenüber seinen Arbeitnehmern als Nebenpflicht des Arbeitsvertrages nach $\S 106$ GewO, § 4 Nr. 7 i. V.m. § 15 Abs. 1 S. 1 ArbSchG 
und $\S \S 241$ Abs. 2, 242 BGB zu. Nach § 106 GewO kann der Arbeitgeber die Arbeitsleistung hinsichtlich des Inhaltes, des Ortes und der Zeit nach billigem Ermessen bestimmen, sodass gewisse Arbeitsschutzmaßnahmen Bestandteil der Arbeitsleistungspflicht sein können [3]. Voraussetzung ist, dass eine gebotene Abwägung zwischen den Arbeitgeberund Arbeitnehmerinteressen erfolgt (stRspr. u. a. BAG NZA 2019, 619).

\section{Merke}

Eine Impfung als Nebenpflicht aus dem

Arbeitsverhältnis ohne geschriebene Rechtsgrundlage

kann verlangt werden, wenn gewichtige Interessen

betroffen sind.

Betreiber von Krankenanstalten und Pflegeeinrichtungen haben ein erhöhtes Schutzinteresse gegenüber ihren Patienten. Einerseits soll die Impfung eigene Arbeitnehmer schützen, anderseits sollen die mit den Arbeitnehmern in Kontakt stehenden Patienten vor schweren Krankheitsverläufen geschützt werden. Als Indiz, ab wann Pflichtimpfungen arbeitgeberseits angewiesen werden können, kann die Priorisierung der Impfberechtigten herangezogen werden [4]. Im Einzelfall kann sich aus der allgemeinen Treuepflicht des Arbeitnehmers eine Impfpflicht ergeben. Allerdings sind der Treuepflicht Grenzen gesetzt, sodass das berechtigte Arbeitgeberinteresse gegenüber den Arbeitnehmerinteressen überwiegen muss, da keine allgemeine Nebenpflicht des Arbeitnehmers zur Durchführung von medizinischen Behandlungen besteht. Insgesamt können Arbeitgeber im medizinischen Bereich auch ohne geschriebene Regelung anordnen, dass sich ihre Arbeitnehmer einer Schutzimpfpflicht zu unterziehen haben. Eine zwangsweise Durchführung einer Impfung ist jedoch unzulässig.

\section{Nachweispflicht über Impfung}

Im medizinischen Bereich kann es von besonderem Interesse sein, dass Arbeitgeber Kenntnis über den Gesundheitsstand ihrer Arbeitnehmer haben, um i. S.v. § 23a S. 1 IfSG u. a. über die Art und Weise der Beschäftigung zu entscheiden. Bei Daten über Impfungen handelt es sich um personenbezogene Informationen i. S.v. Art. 4 Nr. 15; 9 DSGVO; § 26 Abs. 3 BDSG. Die Verarbeitung solcher Daten zur Durchführung eines Arbeitsverhältnisses kann i.S.v. $\S 26$ Abs.1 BDSG und Art.6 Abs.1c DSGVO i. d. R. als erforderlich angesehen werden, wenn eine wirksam vertraglich vereinbarte Impfpflicht besteht [5].

\section{Merke \\ Datenschutzrechtlich zulässig ist, was zur Ausübung von Rechten oder zur Erfüllung arbeitsrechtlicher Pflichten erforderlich ist [6].}

Dies gilt auch bei besonders sensiblen Gesundheitsdaten i. S.v. Art. 4 Nr. 15 DSGVO, die auch Informationen über Impfungen umfassen. Voraussetzung für die Verarbeitung von diesen Daten ist, dass das Informationsinteresse gegenüber den schutzwürdigen Interessen vom Ausschluss der Informationspreisgabe überwiegt [7].

\section{Arbeitsrechtliche Konsequenzen der Impfverweigerung}

Arbeitsrechtliche Konsequenzen können Impfverweigerern drohen, wenn der Impfschutz eine Arbeitspflicht ist. Der Arbeitnehmer ist nach §611a Abs.1 BGB dazu verpflichtet, die vereinbarte Arbeitsleistung zu erbringen, woraus ihm ein entsprechender Beschäftigungsanspruch gebührt. § 23 Abs. 3 IfSG schreibt vor, dass u. a. Krankenhäuser und Arztpraxen sicherzustellen haben, dass die erforderlichen Maßnahmen getroffen werden, um nosokomiale Infektionen und die Weiterverbreitung von Krankheitserregern zu vermeiden. Daraus ergibt sich für medizinisches Personal die Verpflichtung, das Infektionsrisiko weitreichend einzugrenzen und alle erforderlichen Maßnahmen zu treffen, die geschuldete Arbeitsleistung unter zumutbarerer Risikominimierung zu erbringen. Bei medizinischem Personal wird wohl i. d. R. aufgrund der unmittelbaren Kontaktnähe mit Risikogruppen von einer primären Arbeitspflicht auszugehen sein [8]. Verweigert ein Arbeitnehmer die Impfung, ist ihm die Ausübung der Arbeitsleistung i. S.v. §275 Abs. 1 BGB unmöglich, sodass nach $\S 326$ Abs. 1 BGB sein Vergütungsanspruch entfällt. Liegt dagegen bei der Impfverweigerung nur eine Nebenpflichtverletzung aus dem Arbeitsverhältnis vor, ist die Erbringung der Hauptarbeitsleistung möglich, sodass der Vergütungsanspruch gem. § 615S. 1 BGB bestehen bleibt. Lehnt der Arbeitgeber die angebotene Arbeitsleistung ab, gerät er in Annahmeverzug. Als letztes Mittel kann Arbeitnehmern bei Negierung einer wirksamen Impfpflicht gekündigt werden.

Merke

Bei Impfverweigerern kommt vor allem eine verhaltens- oder personenbezogene Kündigung oder eine betriebsbedingte Kündigung in Betracht.

Voraussetzung für eine verhaltensbezogene Kündigung ist, dass eine wirksame Impfplicht besteht und diese trotz vorheriger Abmahnung nicht durchgeführt wurde [9]. Eine personenbezogene setzt voraus, dass dem Arbeitnehmer die erforderliche Eignung und Fähigkeit fehlt, die geschuldete Arbeitsleistung zu erbringen. Dies ist bei medizinischem Personal gegeben, wenn Arbeitnehmer mit Risikogruppen in Kontakt kommen und mangels einer Schutzimpfung eine erhöhte Gefährdung für Dritte darstellen und keine anderweitige Beschäftigungsmöglichkeit des Arbeitnehmers besteht.

Eine betriebsbedingte Kündigung ist zulässig, wenn Arbeitgeber aufgrund unternehmerischer Entscheidungen die Schutzimpfung für die Ausübung medizinischer Tätigkeiten ab einem konkreten Zeitpunkt zur allgemeinen Voraussetzung erklären [10]. Allerdings ist eine solche Kündigung un- 
zulässig, wenn mildere Mittel in Betracht kommen, wie eine Umsetzung oder Versetzung des Arbeitnehmers auf einen Arbeitsplatz, bei dem es an einem unmittelbaren Kontakt mit Risikogruppen fehlt.

\section{Fazit}

Eine generelle COVID-19-Impfpflicht für Arbeitnehmer besteht nicht. Weder existiert eine solche gesetzlich noch ist sie pauschal als vertragliche Verpflichtung für alle Arbeitnehmer zulässig. Die Anordnung einer verpflichtenden Impfung kann aufgrund berechtigter Arbeitgeberinteressen i.R.d. Abwägung zulässig sein. Dies gilt besonders bei unmittelbarem Kontakt mit Risikogruppen. Das Grundrecht des Arbeitnehmers auf körperliche Unversehrtheit und informelle Selbstbestimmung tritt dann hinter den Arbeitgeberinteressen zurück. Bei Tätigkeiten mit unmittelbarem Kontakt mit Risikogruppen kann der Arbeitgeber nach dem Vorliegen einer Impfung fragen. Impfverweigerern können arbeitsrechtliche Konsequenzen wie Lohnausfall, Abmahnung oder Kündigung drohen.

\section{KERNAUSSAGEN}

- In Arbeits- und Tarifverträgen kann eine Impfpflicht im medizinischen Tätigkeitsfeld vereinbart werden.

- Die Anordnung über die Durchführung von ärztlichen Kontroll- und Schutzmaßnahmen ist nicht per se unzulässig.

- Voraussetzung für eine Impfpflicht ist, dass eine gebotene Abwägung zwischen den Arbeitgeberund den Arbeitnehmerinteressen erfolgt.

- Impfverweigerern können Lohnausfall, Abmahnung und Kündigungen drohen, wenn die Durchführung einer Schutzimpfung eine Arbeitspflicht ist.

Zitierweise für diesen Artikel

Dtsch Med Wochenschr 2021; 146: 933-935

Interessenkonflikt

Die Autorinnen/Autoren geben an, dass kein Interessenkonflikt besteht.
Autorinnen/Autoren

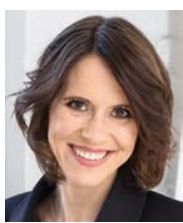

\section{Dr. jur. Klara Bothe, LL.M.}

Studium der Rechtswissenschaft an der Universität Hamburg, LL.M. und Promotion an der Universität Wien, aktuell Rechtsanwältin bei Pochhammer, Rath \& Wellmann Notar und Rechtsanwälte Berlin.

\section{Korrespondenzadresse}

\section{Dr. jur. Klara Bothe, LL.M.}

Pochhammer, Rath \& Wellmann Notar und Rechtsanwälte Kurfürstendamm 123

10711 Berlin

Deutschland

kanzlei@pochhammer-rath-wellmann.de

\section{Literatur}

[1] Naber S, Schulte W. Können Arbeitnehmer zu einer Corona-Impfung oder einem Impfnachweis verpflichtet werden? NZA 2021; 81-82

[2] Benkert D. Wege aus der Pandemie - Impfpflicht im Arbeitsverhältnis? NJWSpezial 2021; 50

[3] Bayer B, Gsellhofer J. Zur Maskenpflicht: Ärztliches Attest vs Maskenpflicht am Arbeitsplatz. ArbR Aktuell 2020; 585-586

[4] Naber S, Schulte W. Können Arbeitnehmer zu einer Corona-Impfung oder einem Impfnachweis verpflichtet werden? NZA 2021; 81-83. m.V.a. §§ 2 ff. CoronalmpfV

[5] Naber S, Schulte W. Können Arbeitnehmer zu einer Corona-Impfung oder einem Impfnachweis verpflichtet werden? NZA 2021; 81-84

[6] Thüsing G, Bleckmann L, Rombey S. Impfpflicht des Arbeitnehmers? COVuR 2021: 66-70

[7] Stück V. Aktuelle arbeitsrechtliche Aspekte zur präventiven und repressiven Bewältigung der viralen Herausforderung. CCZ 2020: 205-208

[8] Thüsing G, Bleckmann L, Rombey S. Impfpflicht des Arbeitnehmers? COVuR 2021: 66-69

[9] Berkowsky W. Die verhaltensbedingte Kündigung - Teil 1. NZA-RR 2001: 1-2

[10] Fuhlrott M, Fischer K. Impfpflicht im Arbeitsverhältnis? NJW 2021: 657-660

online publiziert $\quad 28.05 .2021$

\section{Bibliografie}

\footnotetext{
Akt Rheumatol 2021; 46: 513-516

DOI 10.1055/a-1642-2956

ISSN 0341-051X

(C) 2021. Thieme. All rights reserved.

Georg Thieme Verlag, Rüdigerstraße 14,

70469 Stuttgart, Germany
} 